\title{
Perspective Piece \\ Mobilizing a COVID-19 Contact Tracing Workforce at Warp Speed: A Framework for Successful Program Implementation
}

\author{
Jessica Celentano, ${ }^{1}$ Darpun Sachdev, ${ }^{2}$ Mivic Hirose, ${ }^{2}$ Alexandra Ernst, ${ }^{1}$ and Michael Reid ${ }^{1 \star}$ \\ ${ }^{1}$ University of California, San Francisco, California; ${ }^{2}$ Population Health Division, San Francisco Department of Public Health, \\ San Francisco, California
}

\begin{abstract}
To effectively control the spread of COVID-19, it is essential that all jurisdictions have the capacity to rapidly contact trace all close contacts of each and every case. We describe the early experience in the City and County of San Francisco, where contact tracing capability was rapidly expanded to respond to COVID-19. Important prerequisites to scale up included rapid expansion of the COVID-19 contact tracing workforce, a comprehensive training and onboarding program, and the institution of effective performance management metrics. The San Francisco model for contact tracing, including focusing on rigorous training, recruiting, and partnering with community-based organizations from diverse, affected communities, is an inclusive approach relevant to other jurisdictions and settings.
\end{abstract}

\section{INTRODUCTION}

Despite global consensus on the importance of contact tracing as a critical element of a robust COVID-19 response, ${ }^{1-5}$ the "delivery gap" - that is the gap between an intervention known to be effective and what is actually being delivered-has been profound, most notably in high-income countries such as the United States and Europe. In the United States, for example, only six states have a workforce of contact tracers sufficient to respond effectively to the epidemic. ${ }^{7}$ Closing this "know-do" gap ${ }^{8}$ for COVID-19 has been especially challenging given the urgent need for speed in scaling the response. Efforts to implement contact tracing programs have been exacerbated by a "technical capacity gap," with many public health departments underfunded and understaffed to implement programs of sufficient size or quality. ${ }^{9}$ We describe our early lessons learned from San Francisco, where the Department of Public Health and the University of California, San Francisco, capitalized on decisive public health leadership and expertise in the science of scale $u^{8}$ to rapidly establish a large multidisciplinary contact tracing workforce.

Although the epidemiologic impacts of this workforce have been described elsewhere, ${ }^{10}$ here, we describe 1) how we mobilized a contact tracing workforce, including task-shifting this function to non-public health professionals; 2) training and development, including a unique focus on cultural humility, as an important facet of that workforce's efficacy; 3) the importance of continuous improvement and support to maintaining impact; and 4) the critical role of community-based partners to sustain the impact and ensure that this work reaps a resilient dividend ${ }^{11}$ (Figure 1). Although our insights are most relevant to the specifics of the U.S. public health response to COVID19 , the elements outlined in the following text have relevance to contact tracing programs in diverse settings.

\section{MOBILIZING THE WORKFORCE: IMPORTANCE OF}

\footnotetext{
*Address correspondence to Michael Reid, Institute for Global Health Sciences, University of California San Francisco, 550 16th St., 3rd Floor, San Francisco, CA 94158. E-mail: michael.reid@ucsf.edu
}

\section{TASK SHIFTING}

Being responsive to the need to mobilize sufficient personnel to anticipate a surge in incident cases, the San Francisco Department of Public Health (SFDPH), like many other health departments, rapidly expanded the size of its workforce, including mobilizing more than 270 individuals (108 fulltime equivalents) to conduct COVID-19 contact tracing. Moreover, this expansion happened quickly; in early March 2020, there was no one exclusively focused on COVID-19 contact tracing. By the end of June 2020, the workforce had expanded to more than 150 trained staff and surpassed 250 by September. Determination of the size of the workforce, described elsewhere (M. J. A. Reid et al., in press), was initially informed by epidemiologic modeling and data from other countries and later refined based on recommendations from the $\mathrm{CDC}^{3}$ and internal projections. Most of the initial workforce of contact tracers were furloughed civil servants, from other parts of city government with little to no public health experience. These personnel needed to learn a completely new set of skills as well as new scope of knowledge. Although much of the COVID-19 epidemiology and local guidelines for contact tracing were eminently teachable, ${ }^{12}$ there was early recognition that to be effective, tracers needed to learn how to do the work with compassion, humility, and empathy. As a consequence, training and onboarding focused in large part on refining these softer skills, including the basics of health coaching ${ }^{13}$ and central tenets of delivering client-centered, empowerment-based ${ }^{14}$ care. It included approximately 20 hours of synchronous training, delivered by webinar and online workshops, and included extensive opportunities for practice and skills development. ${ }^{15}$

\section{DEVELOPING THE WORKFORCE: FOCUS ON INCLUSION AND CULTURAL HUMILITY}

The COVID-19 pandemic has revealed profound gaps in the public health infrastructures and exacerbated the inequities that disproportionately affect our most vulnerable populations, making them more susceptible to transmission of COVID-19. In California, higher infection and death rates in Black, Latinx, and Native American communities reveal historical and ongoing legacies of trauma, structural violence and racism, and 


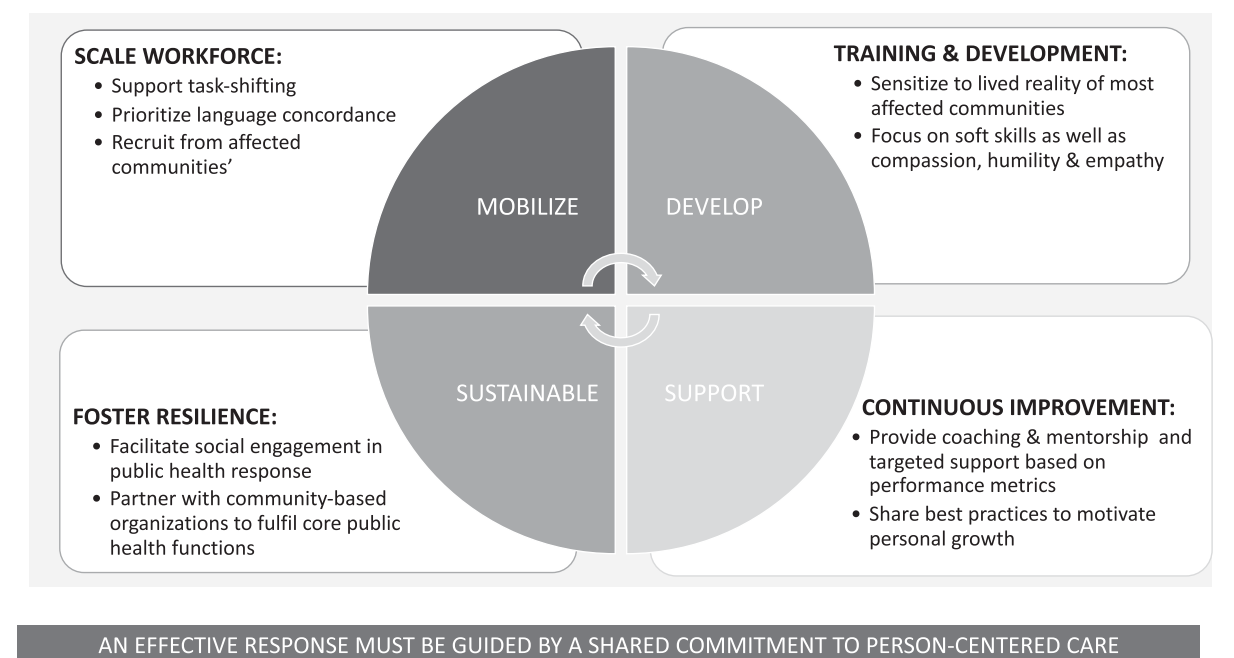

FIGURE 1. Conceptual framework for maintaining a COVID-19 contact tracing workforce.

inequality. ${ }^{16}$ Contact tracing has a crucial role to play in breaking chains of transmission, especially in more affected communities. However, to be maximally impactful, contact tracers need to be aware of and sensitive to the lived reality of members of affected communities. Moreover, contact tracers need to be equipped with the core skills to deliver traumainformed care that is founded on principles of cultural humility, so that they can recognize how their own beliefs and cultural identities might impact the effectiveness of their contact tracing efforts. ${ }^{17}$

Early recognition of the importance of cultural humility to the work of contact tracers led to several interrelated initiatives in San Francisco. First, the SFDPH contact tracing program sought out partnerships with community-based organizations, not only to facilitate buy-in and participation from the communities that those community-based organizations represent but also to ensure that the contact tracing workforce was represented by members of their communities. Second, the program prioritized recruiting a workforce with people who were fluent in the languages of those communities most impacted by COVID-19 in San Francisco, with a goal of ensuring that the language abilities of the workforce matched the preferred languages of the cases and contacts being reached. Third, the program offered additional cultural humility training to all contact tracers to ensure that they were equipped with structural competencies to address the needs of those communities most impacted by COVID-19. ${ }^{18}$ Fourth, the program introduced numerous additional activities to support the physical and mental well-being of the workforce, recognizing that personal wellness and professional resilience were critical to delivering culturally responsive, trauma-informed care.

In addition, the work of the contacting team occurred in tandem with numerous, multisectoral efforts to support those communities most impacted by COVID-19. These efforts included a municipal financial support program, ${ }^{19}$ food and shelter offered to all cases and contacts via the city's COVID19 Department of Operations Center, and a host of programs provided by local charities and community-based organizations. Ensuring that all contact tracers were aware of these different resources and could support cases and contacts to access them was therefore a critical component of the training.

\section{SUPPORTING THE WORKFORCE: ENSURING CONTINUOUS IMPROVEMENT}

Routine review of both process and outcome metrics has been critical for assessing contact tracing successes. By examining these data regularly and matching them with process observations from quality improvement champions, issues and best practices could be identified and rapidly addressed and changes made to contact tracing workflows. Primary measures of success have included the number and percent of new cases and contacts interviewed overall and those interviewed within 24 hours. Related metrics evaluating process outcomes of contact tracing efforts, including the proportion of all contacts who are interviewed, tested, and found to be positive during their 14-day quarantine windows, were also regularly assessed. In addition, the team continuously sought to improve time intervals of each critical process step including time from test performed to positive test result, time from test result to case interview, time from contacts elicited from case to initial interview of contact, and most critically, time from the last exposure to when each contacts went into quarantine. Because the contact tracing effort in San Francisco is still in its nascent stages, the standard operating procedures and the algorithms which inform how contacts are advised about testing and eligibility for quarantine-related resources are being constantly revised, commensurate with evolving science and national guidance. Nonetheless, health equity as central to all elements of the contact tracing response has been crucial. As such, process indicators tracking language concordance between contact tracers and contacts interviewed and aggregate contact tracing performance across different zip codes have been important.

Support for new and inexperienced contact tracers, especially those with minimal public health experience, has also been critical. In San Francisco, individualized coaching is provided to new contact tracers via an intensive onboarding and peer coaching program. This ensures that all new staff understand their roles and responsibilities and can track their 
performance against established standards. Regular tracking of productivity metrics, such as the number of calls made per shift and form completion rates (e.g., demographic variables, referrals to testing and exposure detail variables), is used to help identify when individuals need more training and/or support. Creating interactive communities of practice (CoPs) has also been important in fostering a sense of collaboration and solidarity across a workforce working remotely. Although operations research is ongoing to assess the impact of these CoPs, they represent a highly effective means of rapidly disseminating technical and scientific updates across the workforce.

\section{BUILDING BACK BETTER: ENSURING SUSTAINABLE, RESILIENT RECOVERY}

Although COVID-19 is an unprecedented emergency, it also provides a unique opportunity to build a better health system. We assert that that substantial investment in contact tracing capability should be leveraged to address other public health needs even as the workforce focuses on reaching contacts of those affected by COVID-19. Already the San Francisco contact tracing model, with a critical focus on inclusion and health equity, has been a model for other contact tracing programs elsewhere in California and has catalyzed efforts to standardize cultural humility training across the state. Prioritizing recruitment and retention of a well-trained public health workforce that represents the communities it is serving will reap dividends even after the end of the COVID-19 pandemic.

As community-based organizations are deployed to support contact tracing efforts in San Francisco, like elsewhere, there is also a unique opportunity to leverage the health promotion and community connections of many of those community-based organizations to have an impact that extends well beyond COVID-19. In the near term, this workforce can support local COVID-19 vaccine promotion efforts, assist with demand generation activities, and address vaccine hesitancy concerns. After COVID-19, it is also conceivable that this workforce could help disseminate other public health messages, including promulgating influenza vaccine efforts or connecting expecting mothers to maternal and child health classes. Task-shifting the core functions of contact tracing to community health workers is likely to lead to dividends in how public health departments respond to other diseases and how this essential workforce is leveraged to meet other critical needs of the most vulnerable and most impacted populations of the United States in the future.

\section{CONCLUSION}

The COVID-19 pandemic has led to a dramatic increase in the public health workforce, including mobilizing contact tracers on a scale was inconceivable a few months ago. The San Francisco model for contact tracing-including focusing on rigorous training, recruiting, and partnering from diverse, affected communities-is an inclusive approach relevant to other U.S. jurisdictions. The model also highlights the importance of understanding contact tracing as part of an expansive vision to scale a broader set of public health solutions to control the spread of COVID-19 and build long-term sustainability, especially targeting vulnerable and marginalized populations.
Received December 30, 2020. Accepted for publication February 20, 2021.

Published online April 12, 2021.

Acknowledgments: We acknowledge the hard work of San Francisco's contact tracers whose tireless efforts have been critical to the city's public health response to COVID-19. The American Society of Tropical Medicine and Hygiene has waived the Open Access fee for this article due to the ongoing COVID-19 pandemic.

Authors' addresses: Jessica Celentano, Alexandra Ernst, and Michael Reid, University of California, San Francisco, CA, E-mails: jessica. celentano@ucsf.edu, alexandra.ernst@ucsf.edu, and michael.reid@ ucsf.edu. Darpun Sachdev and Mivic Hirose, Population Health Division, San Francisco Department of Public Health, San Francisco, CA, E-mails: darpun.sachdev@sfdph.org and mivic.hirose@ sfdph.org.

This is an open-access article distributed under the terms of the Creative Commons Attribution (CC-BY) License, which permits unrestricted use, distribution, and reproduction in any medium, provided the original author and source are credited.

\section{REFERENCES}

1. Hellewell $\mathrm{J}$ et al., 2020. Feasibility of controlling COVID-19 outbreaks by isolation of cases and contacts. Lancet Glob Health 8: e488-e496.

2. Fraser C, Riley S, Anderson RM, Ferguson NM, 2004. Factors that make an infectious disease outbreak controllable. Proc Natl Acad Sci U S A 101: 6146-6151.

3. Keeling MJ, Hollingsworth TD, Read JM, 2020. Efficacy of contact tracing for the containment of the 2019 novel coronavirus (COVID-19). J Epidemiol Community Health 74: 861-866.

4. Klinkenberg D, Fraser $\mathrm{C}$, Heesterbeek $\mathrm{H}, 2006$. The effectiveness of contact tracing in emerging epidemics. PLoS One 1: e12.

5. Kretzschmar ME, Rozhnova G, Bootsma MCJ, van Boven M, van de Wijgert J, Bonten MJM, 2020. Impact of delays on effectiveness of contact tracing strategies for COVID-19: a modelling study. Lancet Public Health 5: e452-e459.

6. WHO, 2006. Bridging the "Know-Do" Gap. Meeting on Knowledge Translation in Global Health. Geneva, Switzerland: World Health Organization.

7. Woodsum C, Smith N, Ries E, Chellam M, Chrysler J, Kenney A, Sheng S, Dor H, Fish AJ, 2020. What U.S. States Are Ready To Test \& Trace Today? Available at: https://testandtrace.com/ state-data/. Accessed October 2, 2020.

8. Kruk ME, Yamey G, Angell SY, Beith A, Cotlear D, Guanais F, Jacobs L, Saxenian H, Victora C, Goosby E, 2016. Transforming global health by improving the science of scale-up. PLoS Biol 14: e1002360.

9. Parshley L, 2020. The magnitude of America's contact tracing crisis is hard to overstate. National Geographic. September 1, 2020.

10. Sachdev DD, Brosnan HK, Reid MJA, Kirian M, Cohen SE, Nguyen TQ, Scheer S, 2021. Outcomes of contact tracing in San Francisco, California-test and trace during shelter-inplace. JAMA Intern Med 181: 381-383.

11. Rodin J, 2014. The Resilience Dividend: Being Strong in a World where Things Go Wrong, 1st ed. New York, NY: PublicAffairs.

12. Mooney G, 2016. "A menace to the public health" - contact tracing and the limits of persuasion. $N$ Engl $J$ Med 383: 1806-1808.

13. Huang B, Willard-Grace R, De Vore D, Wolf J, Chirinos C, Tsao S, Hessler D, Su G, Thom DH, 2017. Health coaching to improve self-management and quality of life for low income patients with chronic obstructive pulmonary disease (COPD): protocol for a randomized controlled trial. BMC Pulm Med 17: 90.

14. De Marchis EH, Doekhie K, Willard-Grace R, Olayiwola JN, 2019. The impact of the patient-centered medical home on health care disparities: exploring stakeholder perspectives on current standards and future directions. Popul Health Manag 22: 99-107.

15. Action UPIfEa, 2021. Get Started with Contact Tracing. Available at: https://trailhead.salesforce.com/en/content/learn/trails/getstarted-as-a-contact-tracer. Accessed February 14, 2021. 
16. Azar KMJ, Shen Z, Romanelli RJ, Lockhart SH, Smits K, Robinson S, Brown S, Pressman AR, 2020. Disparities in outcomes among COVID-19 patients in a large health care system in California. Health Aff (Millwood) 39: 1253-1262.

17. Yeager KA, Bauer-Wu S, 2013. Cultural humility: essential foundation for clinical researchers. Appl Nurs Res 26: 251-256.
18. Action UPifEa, 2021. UCSF Pandemic Initiative for Equity \& Action: Training Resources. Available at: https://pandemic.ucsf.edu/ training-resources. Accessed February 14, 2021.

19. Development OoEaW, 2021. Right to Recover Program. Available at: https://oewd.org/employees-impacted-covid-19. Accessed February 14, 2021. 